\title{
Atuação da equipe de Estratégia Saúde da Família no domicílio de pacientes com acidente vascular encefálico
}

\author{
Neiva Junkes Hoepers, M.Sc.*, Ioná Vieira Bez Birollo, M.Sc.*, Luciane Bisognin Ceretta, M.Sc.*, \\ Maria Tereza Soratto, M.Sc.*, Alexandre Machado**
}

${ }^{*}$ Enfermeiras, Discentes do Curso de Enfermagem da Universidade do Extremo Sul Catarinense - UNESC, ${ }^{* *}$ Enfermeiro, Curso de Enfermagem da Universidade do Extremo Sul Catarinense - UNESC

\section{Resumo}

Este estudo de campo teve o objetivo de identificar como se organiza o cuidado domiciliar da pessoa acamada com sequela após acidente vascular encefálico (AVE). Esse tipo de cuidado está cada vez mais assumindo o papel essencial, que inclui assistir com maior comodidade e menores custos pessoas com limitações que as impedem de procurar a assistência ambulatorial. A pesquisa foi de cunho quanti-qualitativo, descritivo e de campo, realizada com 15 pessoas acamadas e com limitaçōes em decorrência de AVE e 15 cuidadores ou familiares, na área de abrangência da Estratégia de Saúde da Família. A coleta dos dados deu-se por entrevistas semiestruturadas, entre março e agosto de 2012, previamente agendadas no próprio domicílio dos pacientes. Os resultados mostraram que o familiar ou o cuidador necessitam estar preparados para cuidar. Verificou-se também a importância da atuação da equipe de saúde, a fim de reduzir complicaçôes clínicas, aumentar a participaçáo da família no cuidado a esse sujeito, com participaçáo na reabilitação, na reintegração social e, consequentemente, proporcionando-lhe uma melhor qualidade de vida. A equipe precisa de reorganização dos serviços e planejamento para visitas domiciliares e orientaçóes específicas para o cuidado das pessoas acamadas com AVE em domicilio.

Palavras-chave: cuidadores, equipe de assistência ao paciente, assistência domiciliar, limitação da mobilidade, atividades cotidianas.

\section{Abstract}

\section{Performance of Family Health Strategy team at home of patients with stroke}

This field study aimed to identify how to organize home care to bedridden person with the sequel stroke. The role of caregiver has been increasingly recognized as an important one, which includes providing care with more comfort and lower costs to people with disabilities who find it difficult to go to outpatient care. This descriptive and field study with quantitative and qualitative approach was carried out with 15 bedridden persons with disability due to a stroke, along with 15 caregivers or family in the Family Health Strategy area. Data collection was performed from March to August 2012 using semi-structured interviews previously scheduled in patients' home. The results showed that the family or 
caregiver needs to be prepared to provide care. Also, we verify the importance of health care team, in order to reduce clinical complications and increase family participation in caring this individual, with participation in rehabilitation, in social reintegration and, thus, providing a better quality of life. The team is in need of services reorganization and of planning home visiting programs and specific guidelines for caring bedridden people with stroke at their home.

Key-words: caregivers, patient care team, home nursing, mobility limitation, activities of daily living.

\section{Resumen}

\section{Actuación del equipo de Estrategia Salud de la Familia en el domicilio del paciente con accidente cerebrovascular}

Este estudio de campo tuvo como objetivo identificar cómo se organiza la atención domiciliaria a persona encamada con secuela después de accidente cerebrovascular (ACV). Este tipo de atención está asumiendo cada vez más un papel muy importante, una vez que incluye mayor comodidad y menores costos para las personas con limitaciones que les impiden buscar atención ambulatoria. Esta investigación cuantitativa y cualitativa, descriptiva y de campo fue realizada con 15 personas encamadas e incapacitadas debido a ACV y 15 cuidadores o familiares, en el área de la Estrategia de la Salud de la Familia. La recolección de datos se realizó, entre marzo y agosto de 2012, a través de entrevistas semiestructuradas previamente programadas en el domicilio de los pacientes. Los resultados mostraron que la familia o el cuidador deben estar preparados para cuidar. Además, se verificó también la importancia de la actuación del equipo de salud, con el fin de reducir las complicaciones clínicas y aumentar la participación de la familia en el cuidado de este individuo, con participación en la rehabilitación, en la reintegración social y, consecuentemente, proporcionándole una mejor calidad de vida. El equipo necesita una reorganización de los servicios y la planificación de las visitas a domicilio y directrices específicas para la atención a personas encamadas con AVE en domicilio.

Palabras-clave: cuidadores, grupo de atención al paciente, atención domiciliaria de salud, limitación de la movilidad, actividades cotidianas.

\section{Introdução}

O acidente vascular cerebral (AVC) ou encefálico (AVE), conhecido popularmente como "derrame cerebral”, é uma doença que consiste na interrupçáo súbita do suprimento de sangue com oxigênio e nutrientes para o cérebro, com déficit neurológico em área cerebral secundária à lesão vascular, lesando células nervosas, o que pode resultar em graves limitaçôes crônicas das atividades, como inabilidade para falar ou mover partes do corpo [1,2].

Embora existam vários tipos de AVE, é possível defini-lo em duas categorias: hemorrágico e isquêmico. A diminuição total ou parcial de fluxo sanguíneo em determinada área do cérebro pode ser definida como quadro isquêmico. Enquanto que o AVE hemorrágico apresenta um sangramento anormal para dentro das áreas extravasculares do cérebro, em consequência de traumas ou aneurismas, levando ao aumento de pressões intracraneanas, resultando em lesóes dos tecidos cerebrais e restringindo o fluxo sanguíneo periférico $[1,3,4]$.

Pessoas com sequelas por AVE seguem normalmente uma rotina de intervenção e tratamento de acordo com o tipo e a causa, que vai do tratamento cirúrgico até o clínico, passando posteriormente para o tratamento fisioterápico. Este consiste, na medida do possível, em reestabelecer funçôes e/ou minimizar sequelas deixadas. No entanto, o quadro que o paciente apresenta - na maioria das vezes, uma hemiparesia ou hemiplegia - dependendo não somente da área cerebral afetada, como também da extensão deste acometimento, tende, com o tempo, a se estabilizar [5].

Desta forma, muitas pessoas que sofrem desse mal acabam tendo sequelas importantes, consequentemente ficando acamados; termo este que pode ser elucidado aos sujeitos que tem sofrido lesão celular cerebral, manifestadas por perdas motoras e da comunicação, distúrbios de percepçáo, perda sensorial, comprometimento cognitivo e efeitos psicológicos, levando a lesóes permanentes que podem ser chamadas de sequela [3]. Ainda, podem comprometer a mobilidade física e resultar em grau de dependência para este indivíduo, principalmente para falar, deambular, ver ou sentir, e incapacitados para exercerem as atividades da vida diária [6]. 
As doenças cerebrovasculares, com destaque para o AVE, são a segunda causa de morte no mundo e a primeira causa de incapacidade funcional entre adultos. Cinco milhóes de pessoas morrem a cada ano por causa de acidentes cardiovasculares [7]. A incidência do AVE é maior após os 65 anos de idade e, considerando o rápido e intenso envelhecimento da população brasileira, estima-se que esse evento seja cada vez mais um relevante problema de saúde pública. Os déficits funcional e cognitivo, além da mudança de personalidade, comportamento e dificuldade para comunicação, são alteraçóes devido ao AVE [8]. Além disso, vem se constituindo como causa principal de internaçóes, mortalidade e incapacidades [9].

O comprometimento da pessoa com sequela por AVE pode apresentar paresia facial; perda de força no braço ou perna de um lado do corpo; alteração súbita da sensibilidade com sensação de formigamento na face, braço ou perna de um lado do corpo; perda súbita de visão num olho ou nos dois olhos; alteraçáo aguda da fala, incluindo dificuldade para articular, expressar ou para compreender a linguagem; dor de cabeça súbita e intensa sem causa aparente; instabilidade, vertigem súbita intensa e desequilíbrio associado a náuseas ou vômitos. Tais manifestaçôes podem ser melhoradas ou reabilitadas com atividades que precisam ser reaprendidas pelo indivíduo dentro da família. Para poder enfrentar a realidade e os novos problemas do cotidiano, o cuidado domiciliar está cada vez mais assumindo importante papel no cuidado, que inclui assistir com maior comodidade e menores custos as pessoas com limitaçôes que os impedem de procurar a assistência ambulatorial [10-12].

Alguns serviços de saúde se adaptam às necessidades do portador de múltiplas comorbidades e de alta dependência, características frequentemente encontradas em pessoas com sequelas por isquemias diversas, entre elas o Acidente Vascular Encefálico (AVE). Tais serviços podem ser chamados de Assistência Domiciliar (AD) [13]. Também é definido pela Resolução de Diretoria Colegiada (RDC) da Agencia Nacional de Vigilância Sanitária (ANVISA), no 11/2006, como um conjunto de atividades de caráter ambulatorial, programadas e continuadas, desenvolvidas em domicílio [14].

Nesse sentido, o Ministério da Saúde (MS), em sua portaria $\mathrm{n}^{\circ} 2.029 / 2011$, institui o Serviço de Atenção Domiciliar (SAD), que vem contribuir para a reorganização do processo de trabalho das equipes que prestam cuidados domiciliares na atenção básica [15]. O SAD fica responsável pelo gerenciamento e operacionalizaçáo das Equipes Multiprofissionais de Atenção Domiciliar (EMAD) e Equipes Multiprofissionais de Apoio (EMAP), pois se caracteriza por um conjunto de açóes de promoçáo à saúde, prevençáo e tratamento de doenças e reabilitaçáo prestadas em domicílio, com garantia de continuidade de cuidados e integrada às redes de atenção à saúde. Além disso, distingue o cuidador como a pessoa, com ou sem vínculo familiar, capacitada para auxiliar o paciente em suas necessidades e atividades da vida cotidiana [15].

Já a Visita Domiciliar (VD) é uma metodologia de trabalho de caráter educacional, assistencial e exploratório, realizada por profissionais que vão ao domicílio do paciente, usuário ou beneficiário de um serviço [16]. O MS, por meio de Portarias aprovadas no Pacto pela Saúde [17], já definia as responsabilidades e açóes estratégias mínimas que todos os municípios brasileiros devem desenvolver no âmbito da Atenção Básica. Dessa forma, a VD e o acompanhamento domiciliar já faziam parte de seu quadro. Para que a VD ocorra, a portaria no $2.527 / 2011$ diz que deve ser desenvolvida por: "equipe multiprofissional e integrada à rede de atenção à saúde; identificar e treinar os familiares e/ou cuidador dos usuários, envolvendo-os na realização de cuidados, respeitando os seus limites e potencialidades; abordar o cuidador como sujeito do processo e executor das açóes; acolher demanda de dúvidas e queixas dos usuários e familiares e/ ou cuidador como parte do processo de Atenção Domiciliar; elaborar reuniôes para cuidadores e familiares; utilizar linguagem acessível a cada instância de relacionamento; promover treinamento pré e pós-desospitalizaçáo para os familiares ou cuidador dos usuários; participar da educação permanente promovida pelos gestores; e assegurar, em caso de óbito [...] emissão do atestado de óbito" [18].

A partir dessas reflexóes, tem-se como objetivo principal deste estudo: Como se organiza o cuidado domiciliar à pessoa acamada com sequela de AVE em Unidades de Estratégias de Saúde da Família em um município do sul de Santa Catarina?

\section{Material e métodos}

O presente estudo possui abordagem metodológica quanti-qualitativa do tipo descritiva e de campo. A pesquisa foi desenvolvida nos domicílios 
dos pacientes acamados e incapacitados por AVE, onde existe cobertura da Estratégia Saúde da Família (ESF). A escolha para o cenário do estudo foram as Unidades Básicas de Saúde (UBS), que possuem os registros de pacientes acamados e incapacitados por AVE, e por meio das quais foi realizado um levantamento do número desses pacientes. Participaram do estudo a pessoa acamada e com sequela de AVE e sua família ou cuidador, compondo uma amostra de 15 pacientes acamados e 15 familiares ou cuidadores, totalizando uma populaçáo de 30 pessoas, de um município do Sul de Santa Catarina, nos meses de maio e junho de 2012. Os critérios de inclusão dos pacientes e seus cuidadores: pacientes acamados e com sequelas por AVE, maiores de 18 anos; cuidadores e ou familiares próximos ou que se fazem mais presentes no cuidado destes pacientes; aceitação para participar da pesquisa a partir da assinatura do Termo de Consentimento Livre e Esclarecido (TCLE).

Os dados foram coletados por meio de entrevistas semiestruturadas, previamente agendadas no próprio domicílio dos pacientes. Foram realizadas duas visitas, sendo a primeira para reconhecimento do cenário e a segunda para aplicação da entrevista.

O estudo foi aprovado pelo comitê de ética e pesquisa da UNESC-SC, CEP no 18095/2012 - CAAE: 02176312.8.0000.0119, e seguiu as exigências formais contidas na resoluçáo 196/96, do Conselho Nacional de Saúde. Para preservar a privacidade e ética dos envolvidos na pesquisa, usaremos a letra "P" seguida de um número para identificar as pessoas que cuidam dos pacientes acamados e com AVE (familiares ou cuidadores).

\section{Resultados e discussão}

Em relação ao perfil dos pacientes acamados com sequela por AVE no domicílio, a faixa etária variou entre 46 a 95 anos, com predominância entre 76 a 85 anos. Quanto ao gênero, houve predominância do sexo feminino. Em relação ao estado civil, a maioria dos pacientes é casada (8), depois os viúvos (4), e os demais se incluem em "outras categorias". No quesito religiáo, encontramos 13 pacientes da religião católica e dois da religiáo evangélica. Em relação à profissão ou situação atual, cinco (5) eram aposentados, quatro (4) pensionistas e seis (6) pertenciam a "outras categorias".

O perfil dos familiares ou cuidadores dos pacientes acamados e com AVE no domicílio, em sua maioria eram familiares (14), destes, oito (8) esposos(as), cinco (5) filhas, uma (1) irmã e somente um (1) cuidador que a família pagava para o cuidado. Isso mostra que cada vez mais a família tem a responsabilidade do cuidado e também a responsabilidade pelas pessoas dependentes, idosos ou com outra sequela que precisa de cuidado.

Atualmente, o cuidado está cada vez mais sendo assumido pelo cuidador, que é a pessoa com ou sem vínculo familiar, capacitada para auxiliar o paciente em suas necessidades e atividades da vida cotidiana e que contribui com as açóes de assistência domiciliar, como a promoção à saúde, prevenção, tratamento de doenças e a reabilitação. No entanto, esses cuidadores precisam de ajuda da equipe de saúde, que tem como uma de suas açóes a visita domiciliar (VD) e que vão ao domicílio do paciente e desenvolvem uma metodologia de trabalho de caráter educacional, assistencial e exploratório [14-16].

Em relação ao aspecto sobre o conhecimento e cuidado do paciente de AVE pelo cuidador, quanto ao tipo de AVE, constatou-se que o cuidador tinha conhecimento sobre o tipo de AVE que o seu paciente acamado tinha sofrido. Dos 15 sujeitos, 12 deles tiveram AVE isquêmico e três hemorrágicos. Dessa forma, percebe-se que quem cuida conhece os tipos de AVE, mas, por outro lado, desconhece a prevenção, tratamento e reabilitação de pessoas com AVE.

No aspecto conhecimento do tratamento utilizado pós AVE, verificou-se que os cuidadores estavam cientes do tipo de tratamento que deveria ser seguido pelos pacientes.

Quando perguntados quanto à prevenção dos riscos de AVE, não souberam responder, como também não souberam informar os medicamentos que faziam uso antes do AVE. Nadruz Junior [19], em seu estudo, aponta que medidas não medicamentosas podem reduzir a pressão arterial e contribuir para a prevençáo do desenvolvimento de hipertensão arterial. Dentre elas, destacam-se a redução no consumo de sal, a realizaçáo de atividade física regular, o controle do peso corporal e adoçáo de um estilo de vida menos estressante.

Em um estudo de análise sobre o conhecimento dos fatores de risco para o AVE, 41,07\% dos entrevistados desconheciam os fatores de riscos, os demais sabiam pelo menos um e poucos conheciam mais de um [20].

Sobre os medicamentos que faziam uso após o AVE, também sabem o que usam, pois são eles, 
os cuidadores, que acabam fazendo a dispensação e administração destes fármacos. Verificou-se que 14 pacientes faziam uso de fármacos e somente um (01) deles não usava nada no momento da pesquisa, mas já usou em outro momento. A maioria deles (8) faz uso contínuo de anti-hipertensivo (Captopril ou Enalapril), os demais, de alguma forma fazem uso de outros medicamentos para melhorar a função cardiocirculatória.

A terapia anti-hipertensiva tem se mostrado mais eficaz na prevenção primária do AVE que na prevenção secundária, sugerindo que outros mecanismos, além da hipertensão, podem estar envolvidos na ocorrência do AVE. Porém, estudo mostra que a terapia medicamentosa anti-hipertensiva desempenha maior proteção contra os infartos lacunares [21].

Em relação ao cuidado dispensado pelo cuidador ou familiar à pessoa com AVE e acamada, apareceram as atividades realizadas no domicílio cotidianamente pelos cuidadores, aquelas que são as chamadas de Atividades de Vida Diária (AVDs): a alimentação, a integridade cutânea, a higiene, as eliminaçôes, a administração medicamentosa, a locomoção e a movimentaçáo da pessoa com AVE. E a maior preocupação dos cuidadores foi a mobilização da pessoa acamada (locomoção e mudança de decúbito). Essas preocupaçôes devem ser consideradas importantes, pois o fato de a pessoa estar acamada significa ter doença que impossibilita de locomover-se, fazendo com que esta permaneça imobilizada, necessitando de cuidados de terceiros.

Independente de haver divisão de tarefas com outras pessoas da família, foi relatada a dificuldade de lidar com o acamado e o cansaço físico ocasionado pelo cuidado contínuo no cotidiano. Estudo acerca das causas que a sobrecarga do cuidado com as pessoas acamadas e com AVE provoca nas famílias mostrou que quem cuida lida com: o grau de dependência física do portador da doença, o isolamento social do cuidador, as mudanças e insatisfaçóes conjugais, as dificuldades financeiras geradas pelo abandono do emprego após a doença e os déficits na saúde física e no autocuidado do cuidador [8]. Mais do que nunca, faz-se necessária a presença do profissional enfermeiro no acompanhamento domiciliar, pois é uma de suas funçôes fazer as intervenções e prescriçóes do cuidado. Como exemplo, o cuidado específico para cada caso e o "auxílio" aos familiares ou ao cuidador para proceder em ocasióes especiais, como as mudanças de decúbito, ou seja, as melhores condutas e as mais indicadas para cada situação, complexas ou náo, que o paciente apresentar. Vale ressaltar que há casos em que este sujeito precisa do acompanhamento, além da equipe de enfermagem e do médico, do fisioterapeuta, do psicólogo, do assistente social e do nutricionista, que nem sempre se fazem presentes na equipe de saúde. Esses profissionais em conjunto podem ajudar o paciente a ter uma reabilitação mais rápida e eficiente, dando condiçóes para que seu estado de saúde se restabeleça mais rápido.

Sobre as orientaçóes que o cuidador recebeu do profissional da saúde, verificou-se que a maioria (67\%) não recebeu orientações, tanto na hospitalização como na UBS. Como podemos ver P1, P2, P4, P5, P6, P7, P11, P12, P13, P15: "não tivemos nenhuma orientação". E somente P8 e P10 receberam orientação na UBS, pela enfermeira e sua equipe. Isso demonstra a falta de orientação na alta hospitalar, como também a importância de se ter a "referência e contra referência", tanto da Atenção Hospitalar como a da Atenção Básica. É de fundamental importância o preparo do paciente e de seus familiares na reorganização da vida em seus lares, de modo que possam assumir os cuidados no domicílio, prevenindo e contornando situaçóes que possam vir a ocorrer [21].

Castro [22] relata que o conhecimento das atividades de cuidado desenvolvidas no domicílio e das dificuldades enfrentadas pelos cuidadores familiares de pacientes incapacitados por AVE poderia contribuir para as atividades educacionais da enfermagem e da equipe interdisciplinar, para que desenvolvam açóes de preparo para pós-alta hospitalar, tais como acompanhamento domiciliário e de atenção ao cuidador, a partir de dados que respondam às necessidades dos cuidadores e dos pacientes acometidos por AVE. Como essas orientaçóes não são transmitidas, os cuidadores acabam cuidando de seus familiares usando os conhecimentos populares: a crença, experiências anteriores, troca de informaçôes com outras pessoas, amigos, vizinhos, grupos ligados à igreja, grupos de voluntários, enfim, a rede de suporte social.

Neste estudo, evidenciou-se que a dificuldade de comunicação é um grande problema para quem cuida, pois os déficits cognitivos resultantes do derrame podem comprometer a capacidade da pessoa de se concentrar, diminuir o nível de atenção, ocasionar a perda da memória de curtos ou longos períodos [23]. 
A alta prevalência de cuidado domiciliar e a provável sobrecarga causada aos familiares, responsáveis pela maioria dos cuidados, são importantes indicadores para o planejamento de açôes que o enfermeiro poderá destinar ao cuidador ou aos familiares. Programas de apoio podem ser dirigidos ao cuidador principal ou mesmo a vários membros da família e podem ser operacionalizados a partir de atendimentos individuais ou em grupo, com trocas de experiências e repasse de orientação prática quanto aos procedimentos de cuidado. Atençáo específica deve ser destinada a indivíduos com as mesmas características daqueles que apresentaram as maiores ocorrências de cuidado domiciliar: idade avançada, baixa escolaridade e incapacidade funcional para atividades instrumentais da vida diária [24].

Observa-se que a maioria das pessoas que cuidam de pessoa acamada e com sequelas de AVE não tem preparo e conhecimento, tanto o básico como o de maior complexidade. Vindo ao encontro do estudo de Castro [22] que relata que as pessoas que cuidam não têm conhecimentos específicos para atender a pessoa acamada, mas aprendem com o cotidiano e diante das dificuldades do atendimento destes pacientes, ou seja, acabam aprendendo gradativamente com as necessidades.

Nesse contexto, nota-se que o conhecimento, informaçóes ou orientaçóes, se foram fornecidas aos cuidadores, não foram suficientes para possibilitar-lhes uma ação segura. Muitas vezes, as orientaçóes incompletas, que se limitam aos "alertas", podem incutir em insegurança, com o que o cuidador poderá deixar de fazer algo que estaria ao seu alcance. Assim, o despreparo do cuidador pode trazer prejuízos ao paciente, resultando em frequentes hospitalizaçóes ou, ainda, piorar as sequelas ou originar outras, consequentemente, ocasionar situaçóes de risco para ambos. Uma das formas de ajuda às famílias em seu processo de cuidar é aquela advinda dos profissionais, que incluem orientaçóes básicas à saúde, e sobre cuidados específicos em casos de doenças crônicas degenerativas, suas complicaçóes e sintomas [25].

Sobre as necessidades dos pacientes e seus cuidadores em relação à atuação de enfermagem da ESF, os cuidadores e pacientes destacaram a necessidade de acompanhamento da equipe de saúde para orientaçôes de atividades específicas dos profissionais, como: verificação da pressão arterial; orientaçôes sobre a situação atual; agendamento para consultas médicas com especialista, como também a consulta pelo médico da unidade, citada por treze (13) deles.
Podemos verificar em algumas das falas a seguir:

"Gostaria que viessem fazer verificação dos sinais vitais, agendamento de consultas". (P1)

"Necessitamos de consulta médica domiciliar, visita da equipe de enfermagem, agendamento de consulta com especialista”. (P2)

"Necessitamos de um acompanhamento com fisioterapeuta, visita domiciliar do médico e verificação da pressáo". (P3)

"Necessitam de consultas médicas domiciliares, orientaçóes sobre a doença do paciente, verificaçáo da pressão, agendamentos de consultas especializadas". (P4)

"Verificação da pressão arterial, visita médica e de enfermagem domiciliar". (P6)

"Consultas médicas domiciliares, ter acompanhamento da equipe de enfermagem para verificação da pressão e as orientaçôes necessárias". (P8)

"Visita domiciliar do médico e da equipe de enfermagem da UBS". (P10)

"Consultas médicas e acompanhamento domiciliar, verificação da pressão”. (P13)

"Necessita de visita médica domiciliar, acompanhamento da equipe de enfermagem para realizar os curativos e verificação dos sinais vitais". (P15)

A principal forma de intervenção profissional para ajudar as famílias no processo de cuidar se verifica por meio da colaboração com orientaçóes básicas de saúde, dos cuidados específicos em casos de doenças crônicas degenerativas e de suas complicaçóes e sintomas. Assim, o paciente e sua família podem vir a alcançar a independência e administrar melhor os cuidados necessários à situação vivida.

Podemos ver que a maior necessidade levantada pelo cuidador e seus familiares ou pacientes desta pesquisa é a Assistência Domiciliar (AD), especificamente a Visita Domiciliar (VD). Para o profissional 
atuar na $\mathrm{AD}$, é necessário o entendimento acerca do significado que a presença do familiar doente no domicílio tem para a família. As pesquisas sobre o assunto exploram conceitos importantes nesse cenário, como cuidador familiar, domicílio, visita domiciliar, rede de apoio social da família e as definiçôes acerca da expressão "assistência domiciliária". Ainda, a proximidade com o sujeito doente tem sido buscada em vários setores da assistência à saúde. Em virtude das características singulares que detém, a assistência domiciliária (AD) destaca-se como modalidade de cuidado que pressupóe competências profissionais ampliadas direcionadas ao sujeito, família e comunidade [26].

A Assistência Domiciliar (AD) é composta por serviços de saúde versátil que se adaptam muito bem às necessidades do portador de múltiplas comorbidades e de alta dependência, características frequentemente encontradas em pessoas com sequelas por isquemias diversas, entre elas o Acidente Vascular Encefálico (AVE), como também entre idosos [13].

Os cuidados prestados pela equipe de enfermagem da UBS, segundo o cuidador e paciente, foram os seguintes:

"Visita de agentes comunitárias" (P1, P3, P11, P14)

"Agendamento de consultas em consultas médicas especializadas" (P1, P3, P8, P9, P10, P12)

$\mathrm{E}$ os demais relataram que não receberam nenhum atendimento ou visita a domicílio, como podemos verificar nas falas seguintes:

"As enfermeiras não fazem nada para a gente e quando pedimos não fazem ou demoram para fazer" (P4).

"Não fazem nada, não realizam nada pela gente" (P5).

"Só agendam consultas, e visita do médico quando pedimos, e só vem na nossa casa quando nós vamos lá pedir, e ainda demoram” (P12).

Então, percebe-se que há falhas em relação à assistência domiciliar e visitas domiciliares. Será que a equipe da ESF não consegue ampliar a abordagem do sujeito para além da dimensão clínica tradicional?

A atenção domiciliar à saúde constitui a modalidade geral da atenção à saúde prestada no domicílio, sendo uma categoria genérica que engloba e representa o atendimento, a visita e a internação domiciliares, cada qual com seus objetivos e características. Tendo como objetivo promover, manter ou restaurar a saúde, maximizar o nível de independência, minimizando os efeitos das incapacidades ou doenças, incluindo aquelas sem perspectiva de cura [27].

Podemos entender que a $\mathrm{AD}$ está diretamente relacionada à atuaçáo da equipe de saúde no domicilio. $\mathrm{O}$ trabalho do enfermeiro no contexto domiciliar consiste em planejar o cuidado, cuidar, ajudar a cuidar, orientar, supervisionar e encaminhar. É preciso conhecer as necessidades de cuidado do paciente, vivenciar as situaçóes e a realidade onde a pessoa está inserida e o grau de dependência para possibilitar a tomada de decisão de como agir. $\mathrm{O}$ desempenho dessa equipe pode estar relacionado ao tempo de permanência e à quantidade de visitas do profissional no domicílio, de acordo com as necessidades da pessoa que precisa e seus familiares.

No item conhecimento dos serviços prestados pela equipe de ESF para atendimento ao paciente e sua família no cuidado domiciliar, os cuidadores e pacientes da pesquisa relataram que não conheciam os serviços que poderiam ser prestados pela ESF. A maioria respondeu que "não sabe quais", relatado por treze (13), e "se tiver, não sabe", relatado por dois (2) deles (P2 e P3).

Embora as UBS desenvolvam trabalho de equipe, também devem trabalhar a interdisciplinaridade dentro da Estratégia de Saúde da Família, já que sua essência é a abordagem da saúde da família, em especial na assistência domiciliar à saúde. Sendo assim, as práticas da $\mathrm{AD}$ pelos profissionais da ESF devem ser repensadas e merecem uma reflexão acerca de sua prática profissional dentro da ESF. Principalmente o enfermeiro, o qual é visto como o gerenciador de toda a equipe.

Mais uma vez, destacamos a importância da Atenção Domiciliar que, segundo o MS e sua portaria no 2029 de 2011, em seu capítulo I, Art. 5, deve seguir as seguintes diretrizes:

"ser estruturado na perspectiva das redes de atenção à saúde, tendo a atenção básica como ordenadora do cuidado e da ação territorial; articular com os outros níveis da atenção à 
saúde, com serviços de retaguarda e incorporado ao sistema de regulação; ser estruturado de acordo com os princípios de ampliação do acesso, acolhimento, equidade, humanização e integralidade da assistência; estar inserido nas linhas de cuidado por meio de práticas clínicas cuidadoras baseadas nas necessidades do usuário, reduzindo a fragmentação da assistência; adotar modelo de atenção centrado no trabalho de equipes multiprofissionais e interdisciplinares; e estimular a participação ativa dos profissionais de saúde envolvidos, do usuário, da família e do cuidador" [15].

Náo podendo deixar de lembrar que a portaria $\mathrm{n}^{\circ} 2.488$, de 21 de outubro de 2011, é que aprova a Política Nacional de Atenção Básica, estabelecendo a revisão de diretrizes e normas para a organização da Atenção Básica, para a Estratégia Saúde da Família (ESF) e o Programa de Agentes Comunitários de Saúde (PACS), com vistas à revisão da regulamentaçáo de implantação e operacionalizaçáo vigentes [28].

Assim sendo, a família tem papel central na condução do cuidado domiciliar. Na prática, é a família que assume a responsabilidade direta pelo cuidado. É ela que primeiro identifica a existência de um problema, que tenta traduzir e elaborar uma explicação para a situação e toma decisóes em relação à melhor forma de conduzi-lo [29]. Não sendo só de sua responsabilidade, pois a equipe de saúde deveria assumir o seu papel, como já descrito anteriormente pelas portarias ministeriais.

\section{Conclusão}

Esta pesquisa possibilitou identificar a assistência que a equipe de saúde da ESF, no município de Criciúma, presta às pessoas incapacitadas por AVE que permanecem acamadas. Além disso, observou-se o trabalho de enfermagem: orientaçóes quanto aos cuidados básicos, realização da visita domiciliar e de grupos operativos.

Verificou-se que os cuidadores desconhecem os serviços prestados pela ESF e que a maior dificuldade que encontram é relacionada às atividades da vida diária.

Portanto, a assistência domiciliária representa uma metodologia de trabalho a ser redescoberta e melhor explorada pelos profissionais. Acredita-se que mais estudos devem ser realizados sobre o assunto, visando à ampliação do conhecimento sobre o AVE e os eventos por ele desencadeados. Os resultados desta pesquisa ficarão à disposição dos profissionais que colaboraram, uma vez que podem contribuir para novas reflexóes a respeito de suas práticas cotidianas nos cuidados aos pacientes com incapacidades decorrentes do AVE, de forma a permitir uma melhor qualidade da assistência prestada a estes indivíduos.

\section{Referências}

1. Winnick JL. Educaçáo Física e Esportes Adaptados. 1a ed. São Paulo: Manole; 2004.

2. Radanovic M. Características do atendimento de pacientes com acidente vascular cerebral em hospital secundário. Arq Neuropsiquiatr 2000;58(1):99-106.

3. Smeltzer SC, Bare BG. Brunner \& Suddarth, Tratado de Enfermagem médico-cirúrgica. 10 a ed. Rio de Janeiro: Guanabara Koogan; 2006.

4. Mazzola D, Polese JC, Schuster RC, Oliveira SG. Perfil dos pacientes acometidos por AVE, assistidos na clínica de fisioterapia neurológica na Univ. de Passo Fundo. Rev Bras Promoç Saude 2007;20(1):22-27.

5. Costa AM, Duarte E. Atividade física e a relação com a qualidade de vida de pessoas com sequelas de acidente vascular cerebral isquêmico (AVCI). Rev Bras Ciênc Mov 2002;10(1):47-54.

6. Andrade LM, Costa MFM, Caetano JA, Soares E, Beserra EP. A problemática do cuidador familiar do portador de acidente vascular cerebral. Rev Esc Enferm USP 2009;43(1):37-43.

7. (OMS) Organização Mundial de Saúde [Internet]. Centro de Mídia; 2008. As dez principais causas de morte. [citado 2012 Mai 28]. Disponível em URL: www.who.int/mediacentre

8. Bocchi SCM, Angelo M. Interaçáo cuidador familiar-pessoa com AVC: autonomia compartilhada. Ciênc Saúde Coletiva 2005;10(3):729-38.

9. Brocchi SCM. Vivenciando a sobrecarga ao vir-a-ser um cuidador familiar de pessoa com acidente vascular cerebral (AVC): uma análise do conhecimento. Rev Latinoam Enferm 2004;12(1):115-21.

10. Brasil. Biblioteca Virtual em Saúde [Internet]. [citado 2013 Mai 1]. Disponível em URL: http://bvsms. saude.gov.br

11. Talarico TR, Venegas MJ, Ortiz KZ. Perfil populacional de pacientes com distúrbios da comunicação humana decorrentes de lesáo cerebral, assistidos em hospital terciário. Rev CEFAC 2011;13(2):330-9.

12. Lessmann JC, De Conto F, Ramos G, Borenstein MS, Meirelles BHS. Atuação da enfermagem no autocuidado e reabilitaçáo de pacientes que sofreram Acidente Vascular Encefálico. Rev Bras Enferm 2011;64(1):198202.

13. Coutinho GC. Instituto Marcos Daniel [online] 2010. [citado 2013 Jun 1]. Disponível em: URL: http: www. institutomarcosdaniel.org.

14. Anvisa. RDC/ANVISA No11, 26 Jan 2006. Dispóe sobre o Regulamento Técnico de Funcionamento de 
Serviços que prestam Atenção Domiciliar. Brasília: Anvisa; 2006.

15. Ministério da Saúde. Port. no 2.029, de 24 agosto 2011. Institui a Atençáo Domiciliar no âmbito do Sistema Único de Saúde (SUS). Brasília: MS; 2011.

16. Teixeira CP. Visita domiciliar: um instrumento de intervenção. Sociedade em Debate 2009;15(1):165-78.

17. Ministério da Saúde. Portaria no 91 de 10 de janeiro de 2007. Pacto pela saúde. Brasília: MS; 2007.

18. Ministério da Saúde. Port. no 2.527, 27 out 2011. Redefine a Atenção Domiciliar no âmbito do Sistema Único de Saúde (SUS), Brasília: MS: 2011.

19. Nadruz Junior W. (AVC) Diagnóstico e tratamento dos fatores de risco. Revista Cidadania e Meio Ambiente; 2009.

20. Silva IHB, Junqueira CM, Cortes PC, Cardoso RF, Marques CCS, Teixeira FCBD, et al. Conhecimento sobre o acidente vascular cerebral em Vassouras-RJ: análise entre população da zona urbana e zona rural. Neurobiologia 2012;75:3-4.

21. Ministério da Saúde. A populaçáo deve ficar atenta aos riscos do AVC. Brasília: MS; 2012.

22. Castro JAB, Epstein MG, Sabino GB, Oliveira GL, Nogueira CB, Staszko KF, Walter Anibal Filho W. Estudo dos principais fatores de risco para acidente vascular encefálico. Rev Bras Clin Med 2009;7:171-3.

23. Perlini NMOG, Faro ACM. Cuidar de pessoa incapacitada por acidente vascular cerebral no domicílio: o fazer do cuidador familiar. Rev Escola Enferm USP 2005;39(2):154-63.

24. Del Duca GF, Thumé E, Hallal PC. Prevalência e fatores associados ao cuidado domiciliar a idosos. Rev Saúde Pública 2011;45(1):1-8.

25. Lacerda MR. Cuidado domiciliar: em busca da autonomia do indivíduo e da família - na perspectiva da área pública. Ciênc Saúde Coletiva 2010;15(5):2621-26.

26. Machado ALG, Silva MJ, Freitas CHA. Assistência domiciliária em saúde: um olhar crítico sobre a produção científica de enfermagem. Rev Bras Enferm 2011;64(2):365-9.

27. Giacomozzi CM, Lacerda MR. A prática da assistência domiciliar dos profissionais da estratégia de saúde da família. Texto Contexto Enferm 2006;15(4):645-53.

28. Ministério da Saúde. Port. no 2.488, 21 out 2011. Aprova a Política Nacional de Atençáo Básica, estabelecendo a revisão de diretrizes e normas para a organização da Atenção Básica, para a Estratégia Saúde da Família (ESF) e o Programa de Agentes Comunitários de Saúde (PACS). Brasília: MS; 2011.

29. Mendes VLF, Molini-Avejonas DR, Ribeiro A, Souza LAPI. A construçáo coletiva de um guia para cuidadores de pacientes acamados: relato de experiência. J Soc Bras Fonoaudiol 2011;23(3):281-7. 